\title{
Editorial \\ The behavioural pharmacology of dementia
}

Behavioural Pharmacology 2017, 28:91-93

\begin{abstract}
Almost 50 million individuals live with dementia, worldwide, at an estimated cost of almost a trillion dollars. In recent years, research has clarified many molecular, ultrastuctural and neurodegenerative processes underlying dementia. Unfortunately, however, this solid progress in understanding the neurobiology of dementia has not as yet been matched by advances in treatment, which still rests largely on the use of drugs that potentiate cholinergic activity in the central nervous system. Such treatments can only slow the symptomatic progress of the disease; they neither ameliorate nor inhibit the underlying pathology.
\end{abstract}

The increase in life expectance, now approaching 80 years, and a concomitant increase in the proportion of elderly individuals (it is predicted that $10 \%$ of the population will be aged 65 and older by 2020) has significant implications for society. For Europe and the Americas, these numbers will be exceeded considerably (85 years and up to $20 \%$ of their citizens). It is therefore timely to explore the current status of the behavioural pharmacology of dementia research, with an overall, but not exclusive, focus on Alzheimer's disease (AD).

$\mathrm{AD}$ is the most common form of dementia and it is estimated that a new case is diagnosed every $3.2 \mathrm{~s}$ worldwide (World Alzheimer Report 2015: https://www. alz.co.uk/research/worldalzheimerreport2015summary.pdf). It is therefore a disease of epidemic proportion, but patients can only be offered symptomatic treatment. An up-todate search at the National Institute of Ageing returned a total of 129 clinical trials currently active in the USA (https://www.nia.nih.gov/alsheimers/clinical-trials/49e9cfc), but there is little hope for an innovative diseasemodifying therapy in the near future. Such a negative view was underlined recently by the failure of a series of clinical trials targeting the amyloid cascade of plaque formation. The latest announcement for the EXPEDITION 3 trial, in which 2100 Alzheimer patients failed to benefit from the monoclonal antibody solanuzemab administered for 80 weeks, questions whether clearance of protein aggregates may ever succeed in modifying disease progression and halting cognitive decay. What follows is the urgent need for novel concepts and approaches.

This Special Issue is in two parts: part one is comprised of a series of reviews on the various dementia models and the potential transmitter systems and intracellular cascades that are compromised during disease progression; part two includes a series of drug-related empirical contributions exploring potential links between inflammation and metabolic diseases and dementia.

To set the scene, Mhillaj and colleagues provide a short introduction to the pathology of $\mathrm{AD}$ and its principal components, amyloid and tau protein, before embarking on a chronologically ordered review of the spontaneous, pharmacological and genetic rodent models. These have led to a significant enrichment of the mechanistic underpinnings of dementia and broadened the options for drug development. Putatively involved neurotransmitters such as glutamate, serotonin, $\gamma$-aminobutyric acid and acetylcholine, and their receptors, are critically appraised and some are followed up in subsequent contributions.

The neurotransmitter identified first in conjunction with memory failure and cognitive decline was acetylcholine. The original cholinergic hypothesis for dementia (and specifically $\mathrm{AD}$ ) was developed over 30 years ago, on the basis of the finding that, in $\mathrm{AD}$ brains analysed post mortem, there is a significant shrinkage of the volume and a loss of more than $50 \%$ of neurons in the basal forebrain that label for the acetylcholine-synthesizing enzyme choline acetyltransferase. As Douchamps and Mathis point out, projections from the basal forebrain richly innervate both the hippocampus and the cortex, and these areas are also compromised in AD. They highlight the emergence of cholinomimetics, acetylcholine esterase inhibitors and muscarinic/nicotinic receptor agonists, which constitute the first, and still predominant, symptomatic therapy for dementia. They further suggest that with a more nuanced approach, there may still be mileage in the cholinergic hypothesis, particularly when considered in tandem with the role of other neurotransmitter systems. Taking this perspective, and given the dominance of acetylcholine esterase inhibitors as the principal drug therapy in dementia, Giménez-Llort and colleagues have begun to explore the utility of molecules that combine moieties of existing treatments. For example, hybridization of tacrine and huperzine A to Huprine $\mathrm{X}$ was significantly more efficient to antagonize amyloid pathology in vitro and in vivo than its component treatments. Moreover, heterodimers of donepezil and huprine maintain their cholinesterase inhibition by simultaneous binding to peripheral and catalytic sites of the enzyme. 
These compounds have the additional advantage of blocking amyloid and improving cognitive performance of otherwise memory-compromised mice. Other hybrids may follow in the future that could prove efficacious as dementia treatment because of their polypharmacology.

Two reviews follow exploring other neurotransmitter systems. Serotonin (5-hydroxytryptamine; 5-HT) and its receptors have experienced a recent revival of interest, given the availability of novel compounds with nanomolar activity for selective receptor subtypes. As highlighted by Meneses, 5- $\mathrm{HT}_{2}$ and $5-\mathrm{HT}_{6}$ receptors are of particular interest as their stimulation enhances neuronal plasticity and memory formation, possibly by preventing the hyperphosphorylation of tau proteins. Some overlap appears to exist with the cannabinoid system, for which cannabidiol has been promoted as a treatment option for dementia. A lowering of oxidative stress and inflammation may be the reason for reduced tau hyperphosphorylation and cognitive recovery that has been reported in animals models of AD. Karl and colleagues have significantly contributed towards this theme and their review focuses on the pharmacology of cannabidiol, which is the second most common cannabinoid of the hemp plant. This phytocannabinoid lacks psychoactivity, but exerts a strong suppression of inflammatory and immune responses in AD. In addition to its impact on these processes, cannabidiol has also been reported to influence other neurochemical cascades through its activity on serotonergic and vanilloid receptors.

The following review has an intracellular focus, namely, on the significance for dementia of protein degradation and excretion as well as homeostatic calcium. Koss and Platt place particular emphasis on the endoplasmic reticulum as the intracellular compartment for the handling of abnormally folded proteins and its capacity for excretion of unfolded proteins. The so-called unfolded protein response is highly active during neurodegeneration and protects neurons from cell death. However, prolonged activation of the unfolded protein response itself fuels inflammation and immune responses to the overall detriment of the neuron. Consequently, the selective interference with initiators of the stress response of the endoplasmic reticulum may have some therapeutic future.

The final review by Caramillo and Echevarria introduces the zebra fish as a novel system for modelling dementia. The potential advantages include rapid breeding and maturation, similarity in genetics and blood-brain barrier, and some behavioural responses that are analogous to human cognition. This includes co-orthologues for genes of familiar AD such as amyloid precursor protein and presenilins so that the amyloid hypothesis is readily testable in fish, as are established and novel treatment targets.
Part two of this Special Issue comprises a compendium of studies related to memory formation and neurodegeneration utilizing a variety of pharmacological and genetic models of dementia. In the first empirical paper, Kumar and Singh elicit an experimental model of dementia by intraventricular administration of streptozotocin and report on cognitive rescue via forskolin, an activator of protein kinase A. This intracellular cascade plays a critical role in synaptic plasticity underpinning cognition and the demonstration of restoration of this function may warrant further experimental work on this pathway. Using a similar approach, intraventricular and intrahippocampal drug administration, O'Hare and colleagues report on a proof-of-principle study, in which preaggregated $\beta$-amyloid or a fragment of $\alpha$-synuclein were seeded to cause memory impairments. Using an operant response task, they recorded alternating lever pressing as a proxy of executive function and the running response rate as an output analogous to agitation. This is a comorbidity of $\mathrm{AD}$ neglected widely in experimental research and, once confirmed, may prove useful for exploring add-on treatments for AD patients with psychosis.

A subsequent series of empirical reports address the subjects of oxidative stress, inflammation and neuroprotection. Chen and colleagues used the cerebral hypoperfusion model in rats to mimic vascular dementia. They confirm significant changes in acetylcholine levels and activity in forebrain, but also a strong increase in measures of oxidative stress (glutathione, superoxide dismutase). Intriguingly, spatial learning impairments in the water maze and biochemical markers were normalized by the long-term oral administration of polyphenol extracts of grape seeds. This ischaemia model also causes frank cell loss in the hippocampus and a widespread inflammatory response. As Naderi and colleagues show, markers such as malondialdehyde and the proinflammatory cytokines interleukin-1 and tumour necrosis factor- $\alpha$ are increased by ischaemia, and microglia are activated. Protection in this model is also provided by the broad-spectrum tetracyclic antibiotic, minocycline. Similarly, cognitive decline and neuroinflammation are reported by Rocha de Lima and colleagues using the vascular model of permanent middle cerebral artery occlusion in mice. Rescue of infarct size and neurological scores was achieved by boldine, an extract of the evergreen Peumus boldus (or Boldu boldus), a shrub or small tree native to Chile and Peru. If these provocative findings are confirmed, significant benefits indeed may arise from the antioxidant and anti-inflammatory properties of this extract.

Many elderly patients in need of surgery suffer from anaesthesia-induced transient cognitive impairment and, for some, anaesthesia induces dementia. In the final contribution, Jiang and colleagues explore postoperative cognitive impairment in 18-month-old mice. They present evidence that this cognitive impairment is associated 
with a decrease in circulating levels of insulin-like growth factor 1 and that systemic administration of insulin-like growth factor 1 before the induction of anaesthesia provides partial protection.

Overall, this Special Issue seeks to stimulate further research into the mechanisms and the therapeutic options of dementia. At the same time, the diversity of the approaches reported here provides a true representation of the complexity of the disease, and the links between metabolic failures or neuroinflammation and dementia constitute upcoming and exciting new areas with the potential for novel and innovative treatments.

Gernot Riedel Jack Bergman Louk Vanderschuren Bart Ellenbroek Paul Willner February 2017 